\title{
Evaluación del rendimiento académico en bioestadística y la competencia disciplinar de pensamiento matemático en estudiantes universitarios
}

\author{
PEDRO CÉSAR CANTÚ MARTÍNEZ * \\ MARTHA ALICIA SANTOYO STEPHANO ** \\ Facultad de Ciencias Biológicas - Universidad Autónoma de Nuevo León \\ Recibido el 21-08-2017; primera evaluación el 31-05-2018; \\ segunda evaluación el 27-07-2018; aceptado el 27-11-2018
}

\section{RESUMEN}

Se reconoce en la enseñanza universitaria como muy importante la estadística para aplicar el rigor de carácter científico. El objetivo de la investigación fue comparar el rendimiento académico en bioestadística y la competencia disciplinar de pensamiento matemático en estudiantes universitarios del área de ciencias biológicas. La investigación es transversal y exploratoria. Se realizó en 187 alumnos de ambos géneros con edades entre 19 a 20 años que cursaron de manera regular la materia. Se halló una asociación significativa entre aquellos que aprobaron el examen de bioestadística y el nivel de pensamiento matemático que ostentaban los estudiantes en sus pruebas de ingreso (valor de $\mathrm{p}<0,01$ ). Ello determinó que si se posee un nivel alto de pensamiento matemático se cuenta con una probabilidad del $90 \%$ de aprobar el examen de bioestadística.

Palabras clave: estadística, pensamiento matemático, estudiantes universitarios

\footnotetext{
* Profesor de la Universidad Autónoma de Nuevo León adscrito a la Facultad de Ciencias Biológicas. Doctorado en Ciencias Biológicas. Miembro del Sistema Nacional de Investigadores en México. Pertenece al Cuerpo Académico 181 de Ciencias Exactas y Desarrollo Humano. Correo: pedro. cantum@uanl.mx

** Profesora de la Universidad Autónoma de Nuevo León adscrita a la Facultad de Ciencias Biológicas. Maestría en Ciencias con especialidad en Enseñanza Superior. Pertenece al Cuerpo Académico 181 de Ciencias Exactas y Desarrollo Humano. Correo: martha.santoyost@uanl.edu.mx
} 
Evaluation of the academic performance in Biostatistics and the disciplinary competence of mathematical thinking in university students

\section{Abstract}

It is recognized in the university education as very important statistic to apply the severety of scientific character. The objective of the research was to compare school performance in biostatistics and competition discipline of mathematical thinking in university students in the area of life sciences. The research is transverse and exploratory. It was 187 students of both genders aged between 19 to 20 years that coursed in a way regular matter. We found a significant association between those who passed the examination of Biostatistics and the level of mathematical thinking that students showed in their admission tests (value of $\mathrm{p}<0.01$ ). Determining if you have a high level of mathematical thinking is boasts a $90 \%$ chance of passing the test of Biostatistics.

Keywords: statistics, mathematical thought, university students.

Avaliaçáo do desempenho acadêmico em bioestatística e competência disciplinar do pensamento matemático em estudantes universitários Resumo

Ele é reconhecido na educação universitária como estatística muito importante aplicar o rigor de caráter científico. $\mathrm{O}$ objetivo da pesquisa foi comparar o desempenho escolar na disciplina de Bioestatística e competição de pensamento matemático em estudantes universitários na área das Ciências da vida. A pesquisa é transversal e exploratório. Foi 187 alunos de ambos os sexos com idade entre 19 e 20 anos que corriam em uma questão de maneira regular. Encontramos uma associação significativa entre aqueles que passaram o exame de Bioestatística e o nível de pensamento matemático que os alunos mostraram em seus testes de renda (valor de $\mathrm{p}<0,01$ ). Determinar se você tem um alto nível de matemática é de pensamento possui 90\% de chance de passar o teste de Bioestatística.

Palavras-chave: estatísticas, pensamento matemático, estudantes universitarios. 


\section{INTRODUCCIÓN}

La estadística es una asignatura que en el medio académico universitario es de suma importancia, por lo cual ha sido agregada a los planes curriculares de distintas carreras profesionales universitarias. De hecho se le reconoce en la sociedad y en la comunidad científica como una herramienta de gran trascendencia para el desarrollo de los avances científicos y tecnológicos, dado que se ha constituido en el medio para «explicar las condiciones regulares en los fenómenos de tipo aleatorio, que componen buena parte de nuestro mundo» (Barreto-Villanueva, 2012, p. 7). De acuerdo con Moya (1989), esta se define como «la rama del saber que trata del desarrollo y aplicación de métodos eficientes de recolección, elaboración, presentación, análisis e interpretación de datos numéricos» (p. 25). Asimismo, Cantú-Martínez y Gómez (2003) indican que la asignatura de estadística puede denominarse de forma diferente en función al campo o disciplina en que se emplea, señalando que «aplicada al campo de la salud, se denominaría estadística de salud, en el área de estudio y caracterización de la población humana, se le llama estadística demográfica, en su parte social como estadística social y en las ciencias biológicas, bioestadística» (p. 2).

No obstante las distintas denominaciones existentes, todas estas aluden a un mismo propósito que es el «reunir una información cuantitativa concerniente a individuos, grupos, series de hechos, etc. y deducir de ello gracias al análisis de estos datos unos significados precisos o unas previsiones para el futuro» (Ruiz, 2004, p. 3).

Reconocida esta relevancia de la estadística en la vida cotidiana, científica y en la formación universitaria, varios investigadores como Estrada, Batanero y Fortuny (2004), Vanhoof, Castro, Onghena, Verschaffel et al (2006), Evans (2007), Mondéjar, Vargas y Bayot (2008), Rodriguez (2011), y Torres, Aparicio, Bazán y Abdounur (2015), se han acercado al estudio de la relación entre la actitud hacia la estadística que manifiestan los estudiantes con su rendimiento académico, como también con sus estilos de aprendizaje y dificultades que encuentran en la materia. Sin embargo, haciendo una búsqueda pormenorizada, no encontramos indagaciones donde se relacione las competencias disciplinares de los estudiantes, particularmente el pensamiento matemático, con su rendimiento académico en bioestadística.

Por lo tanto, nos ha resultado de sumo interés explorar esta relación, esencialmente si en la Universidad Autónoma de Nuevo León se aplica el EXANI-II como instrumento para la elección de los estudiantes que desean ingresar a licenciatura. Este examen nacional para el ingreso a la educación 
superior valora la habilidad de conocimiento e identificación de información, la capacidad de sistematización e integración de la información, como también la competencia de interpretación y aplicación del conocimiento (Ceneval, 2016). Especialmente, el EXANI-II evalúa el pensamiento matemático en el estudiante al examinar «la competencia para comprender y resolver situaciones que implican el uso de estrategias de razonamiento aritmético, algebraico, estadístico y probabilístico, geométrico y trigonométrico» (Ceneval, 2016, p. 12), que deberían poseer en el ámbito del campo matemático los estudiantes de educación media superior que desean incorporarse a una carrera profesional.

El interés en este tópico también parte de la inseparable relación existente de la bioestadística y la investigación científica. Por lo tanto, en este manuscrito mostramos un estudio en el que comparamos el rendimiento escolar en la asignatura de bioestadística y la competencia disciplinar de pensamiento matemático en estudiantes universitarios que proceden de una dependencia universitaria de ciencias biológicas. Se evalúa, asimismo, si existen diferencias entre las variables de estudio en función de la carrera profesional que cursan, del género y si aprobó o no el curso de bioestadística.

\section{MARCO TEÓRICO}

En México, indistintamente de las circunstancias de carácter nacional, la educación sigue siendo un baluarte en el escenario social para impulsar el desarrollo, tanto económico, cultural como político-social, siendo una de las preocupaciones nodales de nuestra sociedad. Esto se torna aún más relevante en el marco del proceso de selección de aspirantes que llevan a cabo algunas universidades, lo que se ve acrecentado por el número de demandas de un espacio para continuar sus estudios superiores. En este rubro, los exámenes de ingreso se constituyen en un instrumento sumamente valioso para garantizar que los aspirantes que accedan a su ingreso cuenten con capacidades y competencias óptimas que garanticen su trayectoria académica sin contratiempos; donde se pone en evidencia «la eficacia de educación escolar precedente al ingreso» (Rodríguez y Gómez, 2010, p.48).

Por lo anterior, la calidad educativa se ha tornado sumamente importante en las instituciones de educación superior en los últimos años (Márquez, 2004; Linarez, 2014), por lo que se ha prestado mucha atención a los procesos de enseñanza aprendizaje. Entre las variables que se relacionan con el marco del proceso de enseñanza aprendizaje, encontramos el rendimiento académico de los estudiantes universitarios; el cual se constituye en «la suma de diferentes 
y complejos factores que actúan en la persona que aprende» (Garbanzo, 2007, p. 46). Al respecto, expresa Edel (2003) que:

Cuando se trata de evaluar el rendimiento académico y cómo mejorarlo, se analizan en mayor o menor grado los factores que pueden influir en él, generalmente se consideran, entre otros, factores socioeconómicos, la amplitud de los programas de estudio, las metodologías de enseñanza utilizadas, la dificultad de emplear una enseńanza personalizada, los conceptos previos que tienen los alumnos, así como el nivel de pensamiento formal de los mismos (p. 2).

Ante lo anterior, podemos inferir que el rendimiento académico que muestran los estudiantes nos refiere a un suceso de carácter multifactorial. Sin embargo, se reconoce que el rendimiento académico puede variar en función, de lo que denomina Lamas (2015) como «circunstancias, condiciones orgánicas y ambientales» (p. 316). Por lo cual se constituye en un campo muy atrayente y complejo.

La complejidad, a la que hacemos referencia, principia cuando al rendimiento académico también se «le denomina como aptitud escolar, desempeño académico o rendimiento escolar, pero generalmente las diferencias de concepto solo se explican por cuestiones semánticas, ya que generalmente, en los textos, la vida escolar y la experiencia docente, son utilizadas como sinónimos» (Edel, 2003, p. 2). Una vez señalado lo anterior, citamos que el «rendimiento académico del estudiante es el resultado de la ejecución de una serie de comportamientos necesarios en el procedimiento didáctico a partir del supuesto de que conducen a los alumnos a aprender lo requerido» (Fuentes, 2004, p. 24). En este sentido, Torres y Rodríguez (2006), conceptualizan el rendimiento académico como lo externado por los estudiantes mediante «el nivel de conocimientos demostrado en un área o materia comparado con la norma (edad y nivel académico), generalmente medido a través del promedio escolar» (p. 262); el cual está fuertemente asociado a las experiencias previas y aptitudes de los propios estudiantes. En estos últimos aspectos, las experiencias y aptitudes, se ven reflejadas por «la habilidad intelectual, referida a que los estudiantes que saben formular hipótesis, generar soluciones y comparar y analizar información, tendrán mejor rendimiento académico que los que se acostumbran a memorizar y reproducir detalles» (Willcox, 2011, p. 2).

En este sentido, la materia de estadística retoma suma importancia, ya que esta nos permite adquirir destrezas prácticas para la comprensión y examen de una considerable cantidad de información, lo que evidencia que la disciplina de la estadística posee un carácter transversal y que puede ser aplicada a otros campos disciplinares (Chirinos, Ramírez y Villegas, 2012). No obstante esta 
relevancia que ostenta la disciplina de la estadística, se ha podido observar que los estudiantes universitarios, por lo general, adolecen de un conocimiento y dominio al respecto de forma significativa; y las complicaciones que sobrevienen están sustentadas en debilidades en el área de matemáticas, que se manifiestan en diferentes niveles cognitivos y matemáticos (Instituto Nacional para la Evaluación de la Educación, 2017).

El Programa Internacional de Evaluación de Estudiantes (PISA) contempla la capacidad de los estudiantes para dominar la dimensión sobre matemáticas como alfabetización matemática o como competencia matemática (Rico, 2006). En México, de acuerdo con los datos proporcionados por la prueba PLANEA 2017, —llevada a cabo por la Secretaría de Educación Públicaexhiben que los alumnos de educación media superior — llamada también preuniversitaria - en el rubro de matemáticas, 66 estudiantes de cada 100 o bien, 2 de cada 3 estudiantes se ubican en el nivel 1, donde ellos ostentan «dificultades para realizar operaciones con fracciones y operaciones que combinen incógnitas o variables (representadas con letras), así como para establecer y analizar relaciones entre dos variables» (Instituto Nacional para la Evaluación de la Educación, 2017, p. 6); es decir, no cuentan con suficientes conocimientos para llevar a cabo deducciones a partir de un modelo matemático.

\section{Metodología}

La presente investigación, de carácter transversal y exploratorio, se desarrolló de junio a octubre de 2016, en estudiantes de la Facultad de Ciencias Biológicas de la Universidad Autónoma de Nuevo León (Nuevo León, México). La muestra fue aleatoria y correspondió a 187 alumnos de ambos géneros con edades entre 19 a 20 años que cursaron de manera regular la materia de bioestadística. Los participantes corresponden a las cuatro carreras que se ofrecen en la dependencia como son Biólogo (BIOL), Químico Bacteriólogo Parasitólogo (QBP), Licenciado en Ciencias de los Alimentos (LCA) y Licenciado en Biotecnología Genómica (LBG). Se recabó información relacionada con su género, carrera cursada y se indagó sus calificaciones de la materia de bioestadística y la correspondiente a la competencia disciplinar de pensamiento matemático, resultante de su examen de ingreso, mediante la consulta en el Sistema Integral para la Administración de los Servicios Educativos de la Universidad Autónoma de Nuevo León, a través del módulo de Tutorías. Con la información recabada se integró una base de datos para ser analizados en el programa estadístico SPSS. 


\section{Resultados}

\subsection{Descripción de la población de estudio}

De los 187 estudiantes considerados para esta investigación, el 28,87\% ( $\mathrm{f}=54$ ) correspondió a la carrera de LCA, siguiéndole la carrera de LBG, BIOL y QBP, con $28,34 \%(\mathrm{f}=53), 21,39 \%(\mathrm{f}=40)$ y $21,39 \%(\mathrm{f}=40)$ respectivamente. Con respecto al género, 63,10\% ( $\mathrm{f}=118)$ fueron mujeres y $36,89 \%(\mathrm{f}=69)$ correspondió a hombres. Mientras que 69,51\% ( $\mathrm{f}=130)$ aprobó el curso de bioestadística y $30,48 \%$ (f=57) no lo aprobó.

\subsection{En relación con la carrera cursada}

Como se puede apreciar en la Tabla 1, el promedio más alto de rendimiento académico en bioestadística recayó en los alumnos de la carrera de QBP, con 76,53 y una variación de los datos con respecto a este valor medio de 12 puntos, además se encontró que el $50 \%$ de la población se halla por arriba de la calificación numérica de 79. También se aprecia ostensiblemente que los estudiantes de la carrera de biología (BIOL) presentaron el desempeño más bajo en promedio, con una calificación numérica de 62,30 y una variación de los datos en función del valor medio encontrado de 22,41 puntos. Conjuntamente, se ubicó que el 50\% de la población se localiza por encima de la valoración numeraria de 70,50 (ver Figura 1).

Asimismo, en la Tabla 1 se muestra que la media más alta de rendimiento con relación al pensamiento matemático lo ostentó los alumnos de la carrera de LBG, con un promedio de 1144,68 puntos con una variación de los datos de 94,54 puntos. Conjuntamente, se observó que el 50\% de la población participante está por encima del valor numérico de 1150 puntos. Se aprecia que los estudiantes de la carrera de biología (BIOL) mostraron el puntaje en promedio más bajo de pensamiento matemático con una calificación promedio de 1018,95 puntos y una variación de los datos de 103,74, y el 50\% de la población se encontró por encima de un puntaje de 1012 (ver Figura 2). $\mathrm{Al}$ evaluar si constan diferencias entre las variables de estudio antes descritas, encontramos que, tras el análisis de ANOVA, en el rendimiento académico de bioestadística por carrera cursada, sí existen diferencias significativas $(\mathrm{p}<0,01)$, el mismo comportamiento se muestra para el pensamiento matemático (ver Tabla 1). 
Tabla 1. Carrera cursada, calificaciones de bioestadística y pensamiento matemático

\begin{tabular}{|c|c|c|c|c|c|c|c|c|}
\hline Área & Calificacion & es de b & oestadí & tica & Calificaciones d & ensam & nto mat & emático \\
\hline Carrera & Media (DS) & Mín & Med & Máx & Media (DS) & Mín & Med & Máx \\
\hline $\begin{array}{l}\text { QBP } \\
(\mathrm{n}=40)\end{array}$ & $\begin{array}{c}76,53 \\
(12,00)^{a}\end{array}$ & 20 & 79 & 90 & $\begin{array}{l}1114,75 \\
(85,59)^{c}\end{array}$ & 940 & 1120 & 1276 \\
\hline $\begin{array}{l}\text { LBG } \\
(\mathrm{n}=53)\end{array}$ & $\begin{array}{c}71,98 \\
(12,98)^{\mathrm{b}}\end{array}$ & 38 & 75 & 98 & $\begin{array}{l}1144,68 \\
(94,54)^{d}\end{array}$ & 940 & 1150 & 1300 \\
\hline $\begin{array}{l}\text { LCA } \\
(\mathrm{n}=54)\end{array}$ & $\begin{array}{c}66,13 \\
(20,41)^{a}\end{array}$ & 19 & 71,5 & 94 & $\begin{array}{c}1058,67 \\
(104,29)^{\mathrm{cdd}}\end{array}$ & 868 & 1072 & 1228 \\
\hline $\begin{array}{l}\text { BIOL } \\
(n=40)\end{array}$ & $\begin{array}{c}62,30 \\
(22,41)^{\mathrm{a}, \mathrm{b}}\end{array}$ & 0 & 70,5 & 94 & $\begin{array}{c}1018,95 \\
(103,74)^{\mathrm{c,d}}\end{array}$ & 868 & 1012 & 1252 \\
\hline
\end{tabular}

Nota: ${ }^{a, c}$ QBP es diferente de; b,d LBG es diferente de; valor de $\mathrm{p}<0,01$. Mín: valor mínimo; Máx: valor máximo. Fuente: datos recogidos para la investigación. Elaboración propia.

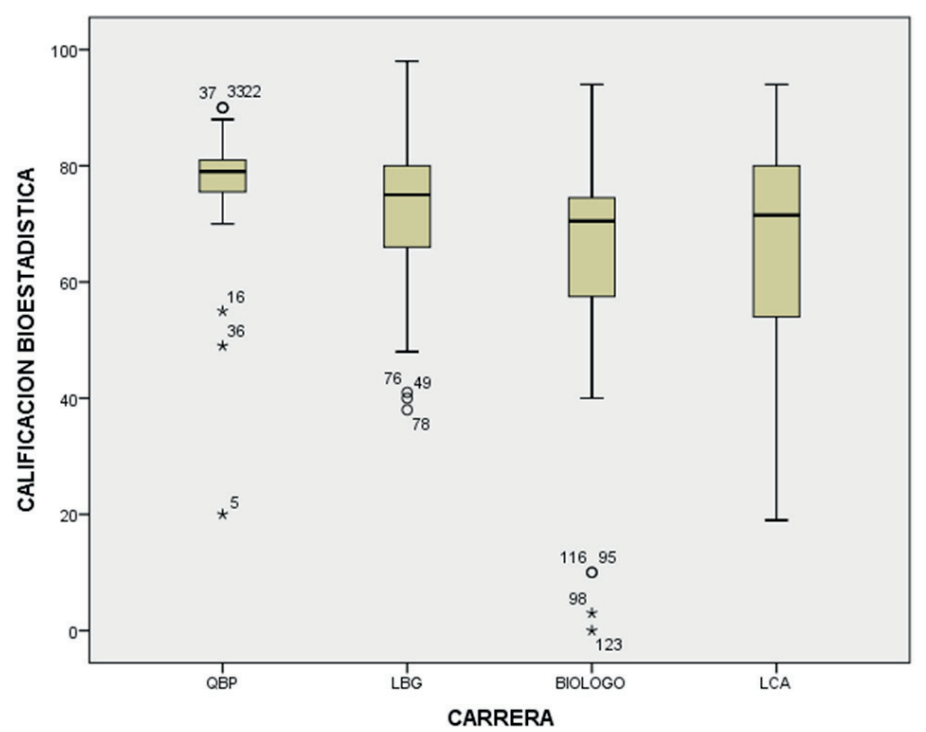

Figura 1. Diagrama de caja y bigotes de las variables calificación de bioestadística y carrera de los estudiantes universitarios.

Fuente: datos recogidos para la investigación. Elaboración propia. 


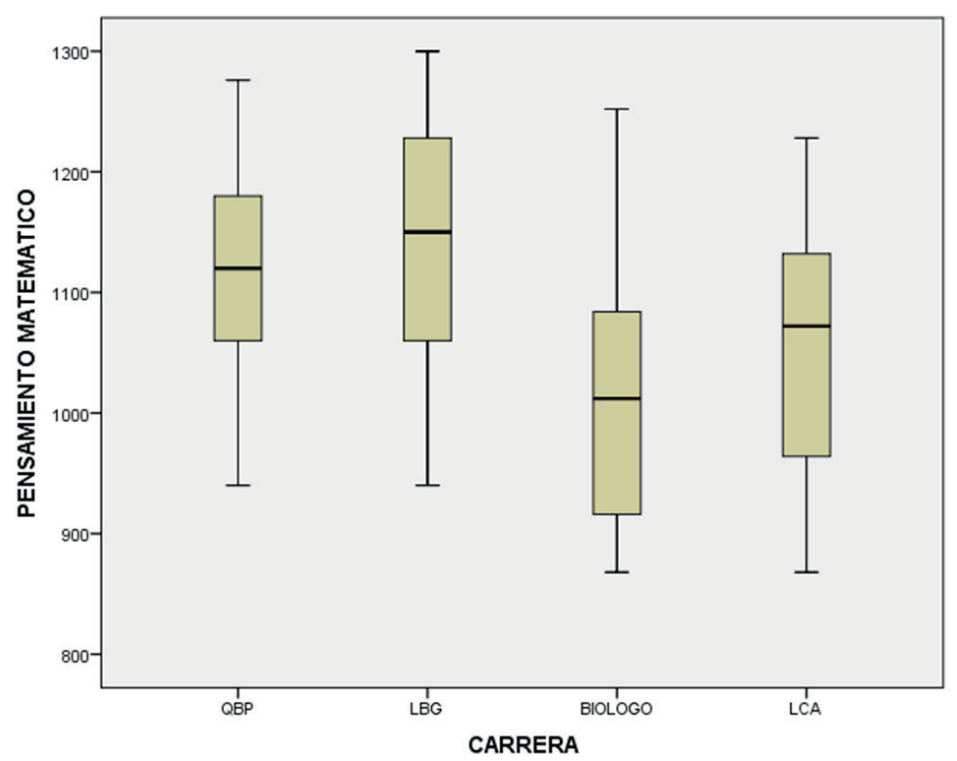

Figura 2. Diagrama de caja y bigotes de las variables pensamiento matemático y carrera de los estudiantes universitarios.

Fuente: datos recogidos para la investigación. Elaboración propia.

\subsection{En correspondencia al género}

Como se puede evaluar en la Tabla 2, el promedio más alto de rendimiento académico en bioestadística se logró en los alumnos de género femenino, con 71,11 y una variación de los datos con respecto a este valor medio de 17,16 unidades. Igualmente, se encontró que el $50 \%$ de la población femenina se encuentra por arriba de la calificación numérica de 75 . Mientras la población estudiada del género masculino cuenta con un valor medio de 65,91 con una variación de los datos de 19,31 puntos. Además, se valúa que el 50\% de la población se encuentra por encima de 72 puntos (ver Figura 3). En tanto que, en lo referente al pensamiento matemático, los varones obtuvieron el valor promedio más alto con 1089,22 puntos con una variación de los datos de 101,21 , y el 50\% de la población masculina estuvo por encima del puntaje de 1108. Por su parte, en el género femenino, el valor medio encontrado para el pensamiento matemático fue de 1084,98 puntos y su desviación estándar de 112,66 puntos, advirtiendo que el 50\% de la población femenina sobrepasó 
el valor de 1084 puntos (ver Figura 4). Al valorar si manifiestan diferencias entre las variables de estudio antes detalladas, encontramos que tras el examen de t Student que no existe diferencia significativa (valor de $\mathrm{p}>0,05$ ) entre género cuando se comparan los resultados de la materia de bioestadística; mismo comportamiento sucede al comparar las calificaciones de pensamiento matemático entre los géneros.

Tabla 2. Género, calificaciones de bioestadística y pensamiento matemático

\begin{tabular}{|c|c|c|c|c|c|c|c|c|}
\hline \multirow{2}{*}{ Género } & \multicolumn{4}{|c|}{ Calificaciones de bioestadística } & \multicolumn{4}{|c|}{ Calificaciones de pensamiento matemático } \\
\hline & Media (DS) & Mín & Mediana & Máx & Media (DS) & Mín & Mediana & Máx \\
\hline $\begin{array}{l}\text { Masculino } \\
(\mathrm{n}=69)\end{array}$ & $\begin{array}{c}65,91 \\
(19,31) \\
\end{array}$ & 0 & 72 & 94 & $\begin{array}{l}1089,22 \\
(101,21) \\
\end{array}$ & 868 & 1108 & 1276 \\
\hline $\begin{array}{l}\text { Femenino } \\
(n=118)\end{array}$ & $\begin{array}{c}71,11 \\
(17,16)\end{array}$ & 10 & 75 & 98 & $\begin{array}{l}1084.98 \\
(112,66)\end{array}$ & 868 & 1084 & 1300 \\
\hline
\end{tabular}

Fuente: datos recogidos para la investigación. Elaboración propia.

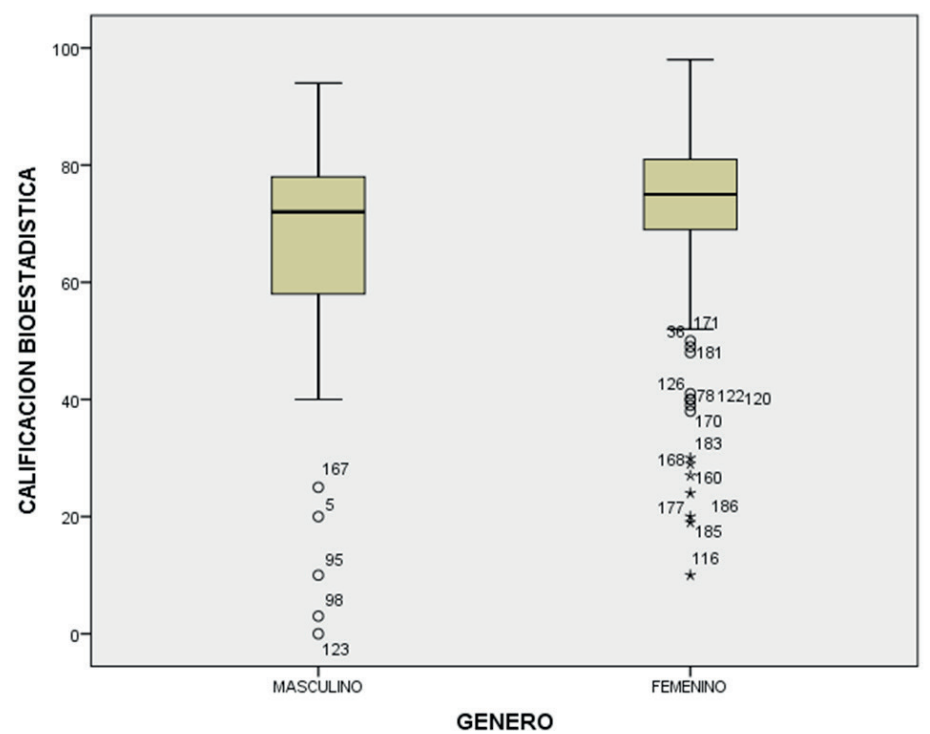

Figura 3. Diagrama de caja y bigotes de las variables calificación de bioestadística y género de los estudiantes universitarios.

Fuente: datos recogidos para la investigación. Elaboración propia. 


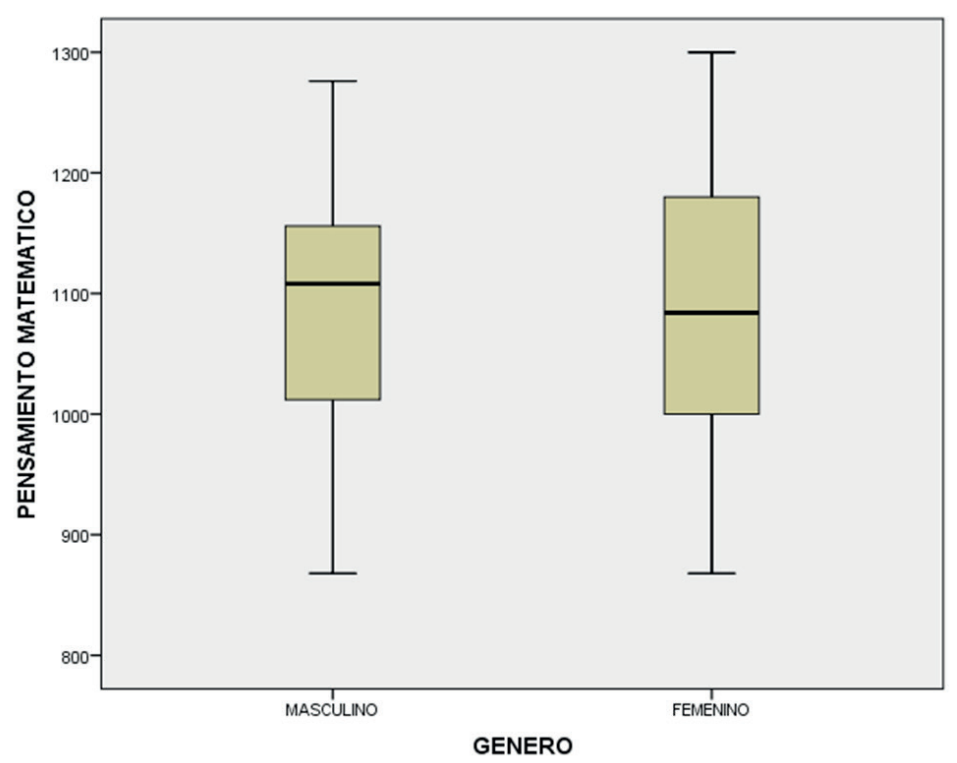

Figura 4. Diagrama de caja y bigotes de las variables pensamiento matemático y género de los estudiantes universitarios.

Fuente: datos recogidos para la investigación. Elaboración propia.

\subsection{En concordancia a la aprobación o no del curso}

Como se logra juzgar en la Tabla 3, el promedio más alto de rendimiento académico en bioestadística se logró en los estudiantes que aprobaron el curso con una media de 78,56 y una variación con respecto a este valor medio de 6,51 puntos. Se encuentra, además, que el $50 \%$ de la población estuvo por arriba de una calificación numérica de 78,5. Mientras la población que no aprobó tuvo un valor medio de 47,82 con una variación de los datos de 17,97 puntos. Además, se apreció que el $50 \%$ de la población se halla por arriba de 54 puntos. Por otra parte, en lo tocante al pensamiento matemático, los que aprobaron el curso contaron con un valor promedio más alto de 1118,33 puntos con una variación de los datos de 93,98 , y el $50 \%$ de la población estuvo por encima del puntaje de 1120. Entre tanto, los alumnos que reprobaron tuvieron un valor medio para el pensamiento matemático de 1013,37 puntos y su desviación estándar de 103,85 puntos, advirtiendo que el $50 \%$ de la población sobrepaso el valor de 1000 puntos. Se encontró diferencia significativa entre aquellos que aprobaron y no, tanto en bioestadística como en pensamiento matemático (valor de $\mathrm{p}<0,01$ ). 
Tabla 3. Aprobó el curso, calificaciones de bioestadística y pensamiento matemático

\begin{tabular}{|c|c|c|c|c|c|c|c|c|}
\hline \multirow{2}{*}{$\begin{array}{l}\text { Aprobó } \\
\text { el curso* }\end{array}$} & \multicolumn{4}{|c|}{ Calificaciones de bioestadística } & \multicolumn{4}{|c|}{ Calificaciones de pensamiento matemático } \\
\hline & Media (DS) & Mín & Mediana & Máx & Media (DS) & Mín & Mediana & Máx \\
\hline $\begin{array}{l}\text { Sí } \\
(n=130)\end{array}$ & $\begin{array}{l}78,56 \\
(6,51)^{\mathrm{a}}\end{array}$ & 70 & 78,5 & 98 & $\begin{array}{l}1118,33 \\
(93,98)^{b}\end{array}$ & 888 & 1120 & 1300 \\
\hline $\begin{array}{l}\text { No } \\
(n=57)\end{array}$ & $\begin{array}{c}47,82 \\
(17,97)^{\mathrm{a}}\end{array}$ & 0 & 54,0 & 69 & $\begin{array}{c}1013.37 \\
(103,85)^{\mathrm{b}}\end{array}$ & 868 & 1000 & 1252 \\
\hline
\end{tabular}

Nota: ${ }^{*}$ El curso se aprueba con una calificación igual o mayor a 70. ${ }^{\mathrm{a}, \mathrm{b}}$ valor de $\mathrm{p}<0,01$.

Fuente: datos recogidos para le investigación. Elaboración propia.

\subsection{Relación entre la aprobación o no del curso de bioestadística y el nivel de pensamiento matemático}

Como se logra observar en la Tabla 4, al realizar la prueba de Ji cuadrada se halló una asociación significativa entre aquellos que aprobaron el examen de bioestadística y el nivel de pensamiento matemático que ostentaban los estudiantes en sus pruebas de ingreso (valor de $\mathrm{p}<0,01$ ). Lográndose determinar que, si se posee un nivel alto de pensamiento matemático, se cuenta con una probabilidad del $90 \%$ de aprobar el examen de bioestadística. Mientras, si se ostenta un nivel moderado y bajo de pensamiento matemático, la probabilidad de aprobarlo disminuyen, siendo del orden de $75,6 \%$ y $41,8 \%$ respectivamente.

Tabla 4. Aprobación del curso o no, y su relación con el nivel de pensamiento matemático

\begin{tabular}{lcccc}
\hline \multirow{2}{*}{$\begin{array}{l}\text { Aprobó } \\
\text { el curso* }\end{array}$} & \multicolumn{3}{c}{ Nivel de pensamiento matemático** } & \multirow{2}{*}{ Total } \\
\cline { 2 - 4 } & Bajo & Moderado & Alto & \\
\hline Sí & 23 & 62 & 45 & 130 \\
No & 32 & 20 & 5 & 57 \\
Total & 55 & 82 & 50 & 187 \\
\hline
\end{tabular}

Nota: ${ }^{*}$ El curso se aprueba con una calificación igual o mayor a 70; ${ }^{* *}$ Puntos de corte: Bajo= menor o igual a 1012 pts.; Moderado= entre 1012 y 1180 pts.; Alto= igual o mayor de 1180 pts.

Fuente: datos recogidos para la investigación. Elaboración propia.

\section{Discusión y RESUltados}

La trascendencia adjudicada, hoy por hoy, a la estadística en la enseñanza universitaria es admitida por la colectividad social, pero de manera más importante 
por los distintos grupos disciplinares que generan el conocimiento y a lo cual se le debe aplicar el rigor de carácter científico en la academia (Barreto-Villanueva, 2012). Con lo antes declarado, se demuestra lo fundamental y valioso que resulta el obtener el conocimiento estadístico con la finalidad de conocer y determinar la probabilidad de ciertos eventos, y con ello, el empleo de la estadística ha conseguido una actuación clave en la investigación científica, siendo así muy relevante en la formación de estudiantes universitarios, aunque esto no sea estimado y aceptado por ellos debido a las dificultades propias de su aprendizaje y que concierne a la misma disciplina. No obstante, en este sentido, Barreto-Villanueva (2012) asevera que «la metodología estadística ha adquirido una importancia tal que el reconocimiento de esta disciplina es un asunto incuestionable en todo el mundo» (p. 11).

Derivado de lo anterior, el conocimiento estadístico posee un papel cada vez más apreciable en la sociedad actual, destacando que el desarrollo de una nación, particularmente en los avances tecnológicos y científicos, tiene correspondencia importante con el empleo de esta disciplina, al hacer «referencia a tendencias en el consumo de diferentes productos, a la distribución de la población y el uso de recursos naturales, entre otros» (Pérez, 2010, p. 3). Esto demarca una gran disparidad entre ostentar y ser competente al emplear el conocimiento científico (Badii, Castillo, Landeros y Cortez, 2007; Pérez, 2010). Consecuente con lo anterior, las Instituciones de Educación Superior deben formar estudiantes con competencias que aborden y respondan a las exigencias actuales, donde hoy en día contar con competencias en el ámbito de la investigación y contar con el conocimiento estadístico se torna pertinente para figurar con una postura crítica frente a la generación y corroboración del nuevo conocimiento científico.

Esto se acentúa más en una dependencia universitaria en el área de las ciencias biológicas que se caracteriza por enfrentar los desafíos actuales del desarrollo científico y la preparación de nuevos profesionales de las ciencias biológicas que respondan a la dinámica y requerimientos del presente. Por lo tanto, «la educación estadística es indispensable en el estudio de fenómenos complejos, en los que hay que comenzar por definir el objeto de estudio y las variables relevantes, tomar datos de las mismas, interpretarlos y analizarlos» (Sánchez y Berenguer, 2013, p. 150), con la finalidad de hacer transitar a los estudiantes por una cultura estadística inicialmente, pasando posteriormente por el razonamiento estadístico y finalmente aproximarse al pensamiento estadístico que es lo deseado. Es importante resaltar que el estudiante debe comprender los principios de la estadística, herramienta multidisciplinar, para «no caer en el supuesto que el ordenador, resuelve la enseñanza de la estadística» (Pérez, 2010, p. 7). De esta forma, los estudiantes lograrán aplicar este conocimiento en otros 
ámbitos, recordando que el «antecedente (expresión) y consecuente (significado) de una función semiótica no se restringen a conceptos, sino abarca toda la anterior ontología de objetos matemáticos u organizaciones de estos en entidades más complejas como sistemas conceptuales o teorías» (Arteaga, 2009, p. 9).

En la presente investigación, se valoró el rendimiento académico de los estudiantes en la materia de bioestadística como también la competencia disciplinar de pensamiento matemático que los estudiantes poseían, observándose que los alumnos de QBP y LBG se constituyen como una población disímil a los estudiantes de LCA y BIOL que tienen un comportamiento equivalente. Por otra parte, se comprobó que hay una diferencia significativa en pensamiento matemático entre aquellos estudiantes que aprobaron o no el curso de bioestadística. Tocante a la perspectiva de género, existe similitud entre hombres y mujeres en relación al rendimiento académico en la materia de bioestadística y las valoraciones que ostentaron de pensamiento matemático. En este último aspecto, un estudio realizado en estudiantes universitarios por Echavarri, Godoy y Olaz (2007) advierten que hay «diferencias estadísticas significativas que permiten afirmar que el rendimiento académico de las mujeres es mayor que el de los varones» (p. 324) de manera general. En nuestro estudio, no obstante haber obtenido las mujeres un mejor rendimiento en promedio en las variables de estudio sobre los hombres, esto no fue significativo estadísticamente. Por otra parte, se encontró una asociación significativa estadísticamente en los alumnos que aprobaron el examen de la materia de bioestadística y el nivel de pensamiento matemático que exhibían los estudiantes universitarios en sus pruebas de incorporación a la universidad. En este sentido, Cortés y Palomar (2008) señalan que «el resultado de una tarea es el mejor predictor para los resultados de esta misma en el futuro, ya que es un reflejo de la concurrencia de numerosos factores como la aptitud del alumno, su voluntad de esfuerzo, las características de la enseñanza que ha recibido» (p. 201).

Por lo tanto, en el presente estudio, el rendimiento académico anteriormente evaluado, de pensamiento matemático se erige en un precedente a considerar que comprobará la razón del rendimiento futuro en la materia de bioestadística.

\section{REFERENCIAS BIBLIOGRÁFICAS}

Arteaga, P. (2009). Análisis de gráficos estadísticos elaborados en un proyecto de análisis de datos. (Tesis inédita de máster). Universidad de Granada, Granada, España. en la investigación científica. Innovaciones de Negocios, 4(1), 107-145. 
Barreto-Villanueva, A. (2012). El progreso de la estadística y su utilidad en la evaluación del desarrollo. Papeles de Población, 18(73), 1-31.

Cantú-Martínez, P.C. y Gómez, L.G. (2003). El valor de la estadística para la salud pública. RESPYN Revista Salud Pública y Nutrición, 4(1), 1-7.

Ceneval (2016). Guía del examen nacional de ingreso a la educación superior (EXANI-II). México: Centro Nacional de Evaluación para la Educación Superior, A.C.

Chirinos, A., Ramírez, O. y Villegas, Z. (2012). Estrategia didáctica para el aprendizaje del contenido estadística descriptiva, en el primer año del L.B. «Luisa Cáceres de Arismendi», ubicado en el municipio Miguel Peña, Valencia estado Carabobo. Revista Ciencias de la Educación, 22(39), 15-37.

Cortés, A. y Palomar, J. (2008). El proceso de admisión como predictor del rendimiento académico en la educación superior. Universitas Psychologica, 7(1), 199-215.

Echavarri, M., Godoy, J.C. y Olaz, F. (2007). Diferencias de género en habilidades cognitivas y rendimiento académico en estudiantes universitarios. Universitas Psychologica, 6(2), 319-329.

Edel, R. (2003). El rendimiento académico: concepto, investigación y desarrollo. REICE Revista Iberoamericana sobre Calidad, Eficacia y Cambio en Educación, 1(2), 1-15.

Estrada, A., Batanero, C. y Fortuny, J.M. (2004). Un estudio comparado de las actitudes hacia la estadística en profesores en formación y en ejercicio. Enseñanza de las Ciencias, 22(2), 263-274.

Evans, B. (2007). Student Attitudes, Conceptions and Achievement in Introductory Undergraduate College Statistics. The Mathematics Educator, 17(2), 24-30.

Fuentes, T. (2004). El estudiante como sujeto del rendimiento académico. Sinéctica Revista Electrónica de Educación, 25, 23-27.

Garbanzo, G.M. (2007). Factores asociados al rendimiento académico en estudiantes universitarios, una reflexión desde la calidad de la educación superior pública. Revista Educación, 31(1), 43-63. https://doi.org/10.15517/ revedu.v31i1.1252

Gorina, A. y Alonso, I. (2013). Concepción de una competencia estadística para el estudiante de doctorado en Ciencias Pedagógicas En J.M. Contreras, G.R. Cañadas, M. M. Gea y P. Arteaga (eds.), Actas de las Jornadas Virtuales en Didáctica de la Estadística, Probabilidad y Combinatoria (pp. 149-156). Granada: Departamento de Didáctica de la Matemática de la Universidad de Granada.

Instituto Nacional para la Evaluación para la Educación (2017). Planea. Resultados nacionales 2017 - Educación Media Superior/Lenguaje y ComunicaciónMatemáticas. México: INEE. 
Lamas, H.A. (2015). Sobre el rendimiento escolar. Propósitos y Representaciones, 3(1), 313-386.

Linarez, G. (2014). Calidad en las instituciones de educación superior en México: ¿utopía o desafío? Revista Iberoamericana para la Investigación y el Desarrollo Educativo, 12(enero-junio), 1-11.

Márquez, A. (2004). Calidad de la educación superior en México. ¿Es posible un sistema eficaz, eficiente y equitativo? Las políticas de financiamiento de la educación superior en los noventa. Revista Mexicana de Investigación Educativa, 9(21), 477-500.

Mondejár, J., Vargas, M. y Bayot, A. (2008). Medición de la actitud hacia la estadística. Influencia de los procesos de estudio. Revista Electrónica de Investigación Psicoeducativa, 6(3), 729-748. https://doi.org/10.25115/ ejrep.v6i16.1303

Moya, L. (1989). Introducción a la estadística en salud. San José: Ed. Universidad de Costa Rica.

Pérez López, C.G. (2010). La estadística como herramienta en la investigación psicológica: un estudio exploratorio. (Tesis inédita de máster). Universidad de Granada, Granada, España.

Rico, L. (2006). La competencia matemática en PISA. PNA, 1(2), 47-66.

Rodríguez, M.P. y Gómez, V.M. (2010). Indicadores al ingreso en la carrera de medicina y su relación con el rendimiento académico. Revista de la Educación Superior, 29(153), 43-50.

Rodríguez, N. (2011). Actitudes de los estudiantes universitarios hacia la estadística. INTERDISCIPLINARIA Revista de Psicología y Ciencias Afines, 28(2), 199-205.

Ruiz, D. (2004). Manual de Estadística. Málaga: Ed. Eumed.net

Torres, F., Aparicio, A. S., Bazán, J.L., Abdounur, O.J. (2015). Actitudes hacia la estadística en universitarios del área de las ciencias de una universidad pública de Chile. Educação Matemática Pesquisa, 17(1), 45-73.

Torres, L.E. y Rodríguez, N.Y. (2006). Rendimiento académico y contexto familiar en estudiantes universitarios Enseñanza e Investigación en Psicología, 11(2), 255-270.

Vanhoof, S., Castro, A. E., Onghena, P., Verschaffel, L., Van Dooren, W. y Van den Noortgate, W. (2006). Attitudes toward statistics and their relation with short- and long-term exam results. Journal of Statistics Education, 14(3), 1-17. https://doi.org/10.1080/10691898.2006.11910588

Willcox, M. del R. (2011). Factores de riesgo y protección para el rendimiento académico: un estudio descriptivo en estudiantes de psicología de una universidad privada. Revista Iberoamericana de Educación, 55(1), 1-9. 